Research Article

\title{
The Electrophysiology of Atrial Fibrillation: From Basic Mechanisms to Catheter Ablation
}

\author{
Panagiotis Ioannidis (iD, Theodoros Zografos $(\mathbb{D}$, Evangelia Christoforatou, \\ Konstantinos Kouvelas, Andreas Tsoumeleas, and Charalambos Vassilopoulos
}

Heart Rhythm Center, Athens Bioclinic, Athens, Greece

Correspondence should be addressed to Panagiotis Ioannidis; ioannidis.pan@gmail.com

Received 23 July 2020; Revised 11 April 2021; Accepted 27 May 2021; Published 7 June 2021

Academic Editor: Robert Chen

Copyright ( 92021 Panagiotis Ioannidis et al. This is an open access article distributed under the Creative Commons Attribution License, which permits unrestricted use, distribution, and reproduction in any medium, provided the original work is properly cited.

\begin{abstract}
The electrophysiology of atrial fibrillation (AF) has always been a deep mystery in understanding this complex arrhythmia. The pathophysiological mechanisms of AF are complex and often remain unclear despite extensive research. Therefore, the implementation of basic science knowledge to clinical practice is challenging. After more than 20 years, pulmonary vein isolation (PVI) remains the cornerstone ablation strategy for maintaining the sinus rhythm (SR). However, there is no doubt that, in many cases, especially in persistent and long-standing persistent AF, PVI is not enough, and eventually, the restoration of SR occurs after additional intervention in the rest of the atrial myocardium. Substrate mapping is a modern challenge as it can reveal focal sources or rotational activities that may be responsible for maintaining AF. Whether these areas are actually the cause of the AF maintenance is unknown. If this really happens, then the targeted ablation may be the solution; otherwise, more rough techniques such as atrial compartmentalization may prove to be more effective. In this article, we attempt a broad review of the known pathophysiological mechanisms of $\mathrm{AF}$, and we present the recent efforts of advanced technology initially to reveal the electrical impulse during $\mathrm{AF}$ and then to intervene effectively with ablation.
\end{abstract}

\section{Introduction}

The need for electrophysiological interpretation of atrial fibrillation $(\mathrm{AF})$ is a perpetual challenge. In recent years, there has been tremendous progress in understanding the pathophysiological mechanisms of AF with the gradual analysis of the seemingly chaotic fibrillatory rhythm. Many times, on this long scientific journey, empirical therapeutic intervention preceded and the recognition or the supposition of the underlying mechanism followed. One of the most basic clinical conclusions, which has been demonstrated in practice by numerous clinical studies, is that the wide and, as much as possible, permanent pulmonary vein isolation (PVI) is the cornerstone for maintaining the sinus rhythm (SR). However, the observation that AF, especially the persistent clinical type, can be maintained in patients with electrically isolated PVs directly raises the question of what should be the next step in the ablation process. In this article, we attempt to review the basic AF electrophysiological mechanisms and the treatment options in catheter ablation.

1.1. Historical Background. The first hypothesis about the mechanism of AF began in the early twentieth century, when Sir Thomas Lewis, having the electrocardiogram and the venous pulse recording as the only observational tools, suggested that $\mathrm{AF}$ was more complicated than the circus movement of atrial flutter [1]. In 1959, Moe described the multiple wavelet hypothesis in which he extended the theory of reentry to include multiple circuits that occur simultaneously and are self-sustaining [2]. According to this theory, the maintenance of $\mathrm{AF}$ is achieved due to the presence of a critical number of reentrant wavelets in the atria, which must have a sufficient mass of myocardium and special characteristics in terms of conduction velocity and refractory period. The multiple wavelet hypothesis was further 
supported by the application of a virtual computational AF model in which the continuous motion of the wavelets (15 to 30 according to the model) showed self-preservation properties in a similar way as in the clinical AF [3]. Moe et al. showed in this model that the probability of simultaneous cessation of all the wavelets is low and that this fact contributes to the self-preservation of AF. If the number of the wavelets becomes less than a critical limit, then there is a greater chance of their simultaneous disappearance and, consequently, the AF termination. Several years later, Allessie et al. provided experimental data supporting the multiple wavelet theory [4]. In isolated dog hearts, using a Langendorff preparation, it was shown with simultaneous recording from 192 points in the atrial myocardium that the AF could be maintained by 3-6 wavelets rotating simultaneously. More supportive evidence in this theory was provided by experimental evidence in similar models, where it appeared that by administering antiarrhythmic drugs, the termination of AF was facilitated by the reduction in the number of reentrant wavelets $[5,6]$. Cox et al. mapped similar electrical activity during human heart surgery, laying the theoretical basis for performing Cox-maze surgery, which had excellent results in AF prevention [7]. It was then widely accepted that multiple mechanisms could play a role in initiating and maintaining AF. Triggers, especially from PVs, can initiate AF and a vulnerable substrate can maintain arrhythmia [8]. Furthermore, as AF is maintained, through the process of remodeling electrical and structural changes in the atrial myocardium can alter the responsible pathophysiological mechanisms [9].

1.2. Functional Reentry and the Leading Circle Model. The simplest form of the functional reentry can be described by the leading circle model, which was first introduced by the French electrophysiologist Allessie in 1977 [10]. According to this model, there is a continuous rotation of the impulse in the periphery of a cycle with simultaneous propagation both peripherally and toward the center of the circle. The impulses directed to the center collide with each other at the same time, making this area refractory. In this way, the impulse is constantly moving around a central, functionally refractory area. This central region in the leading circle concept is the analog of an anatomical barrier (e.g., a scar) which, as we know, can produce a typical, anatomical reentrant circuit. In the typical reentry, the time of a complete rotation is usually longer than the refractory period of the myocardial cells in that area. This means that there is an excitable gap, that is, a time window in which the impulse can enter the circuit finding excitable tissues. If the time of a complete rotation is equal to the refractory period, there is no excitation gap. In functional reentrant circuit such as that of the leading circle, by definition, there is no excitation gap. If the rotation time becomes shorter than the refractory period, then the head of the wavefront will collide into its refractory tail, and the circuit will be terminated (Figure 1). Thus, a functional reentrant circuit without an excitable gap occupies the smallest possible space and rotates at the highest possible frequency, suppressing other potential circuits while having self-sustaining properties.

The multiple wavelets hypothesis and the leading circle concept are complementary, as there is a direct correlation between the cycle length of each circuit, the area it occupies, and the size of the atria. Large atria and reentrant circuits in areas with a short refractory period increase the chance of $\mathrm{AF}$ induction and maintenance. In contrast, atria that are small or have been compartmentalized by ablation or maze surgery cannot easily maintain AF. These theories are also confirmed by clinical observations showing AF increases in patients with left atrial dilatation [11], interatrial and intraatrial conduction disorders $[12,13]$, and pathologically short refractory periods [14]. However, as can be seen from experimental data, the leading circle model is a simplified approach to the complexity of AF maintenance mechanisms.

1.3. The Drivers of AF. Although AF appears to be a chaotic and disorganized rhythm, mapping studies have shown that there are areas with high-frequency depolarization that can be considered as drivers. In these areas, there is a considerable organization, although their activity may spread to the rest of the atrial myocardium in a chaotic and irregular manner, which is characterized as fibrillatory conduction [15]. Mansour et al. [16] in a Langendorff preparation, from sheep hearts, showed that the depolarization frequency in AF introduced with rapid atrial pacing and acetylcholine is much higher in the left atrium than in the right. Consequently, there is a significant frequency gradient from left to right atrium. Areas of dominant frequency are located in the left atrium and in particular in the posterior wall adjacent the PVs. The frequency of depolarization in the left atrium increased with acetylcholine infusion, apparently due to shortening of the action potential, while remaining unaffected after ablation of the interatrial pathways (Bachmann's bundle and inferoposterior pathway). These data suggest that the driving areas of AF are basically located in the left atrium. Of course, whether or not all high-frequency areas represent AF drivers is something that has not yet been clarified.

1.4. PVs and Extra-PV Triggers. The evidence that the dominant frequency is located in the left atrium could be linked with the clinical observation of Michel Haïssaguerre that AF may be initiated by triggers from the PVs [8]. Thus, a significant interest was developed in the pathophysiological role of PVs in AF. Figure 2 shows the possible mechanism a PV trigger can start rotational activity maintaining AF [17].

There are many reasons for the arrhythmogenic activity of PVs. The PV myocardial cells have a higher concentration of slowly and rapidly activating delayed rectifier potassium channels (IKs and IKr) and a lower concentration of cardiac transient outward potassium current (Ito) and L-type calcium channels than in the rest of the atrial myocardium. This setting is responsible for the hyperpolarization of the resting membrane potential and the shorter duration of the action potential, factors that may favor arrhythmogenesis through reentry and triggered activity [18]. 


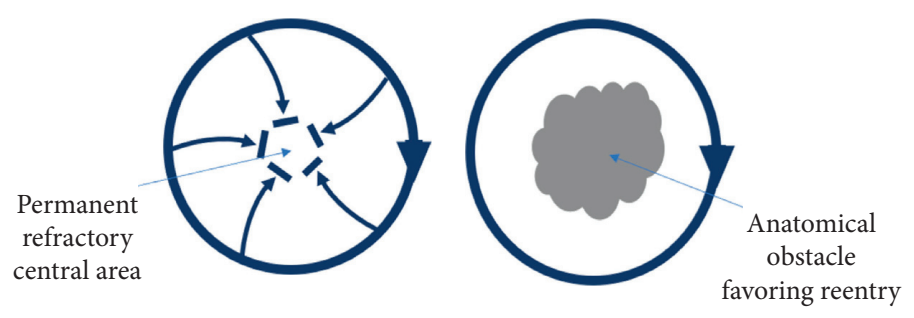

(a) (b)

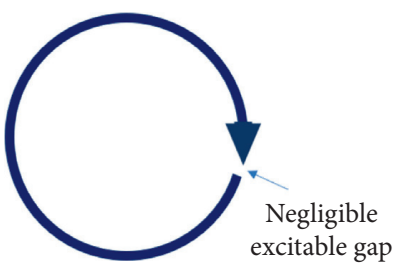

(d)

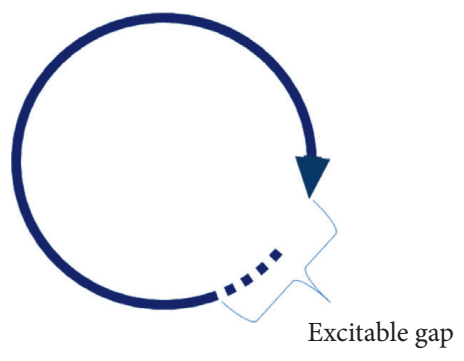

(c) (b) Typical reentrant circuit around an anatomical barrier. (c) Reentrant circuit with the excitation gap. (d) Reentrant circuit without the excitation gap. (e) Unsustainable reentrant circuit as its frontal part collides with its nonexcitable terminal part.

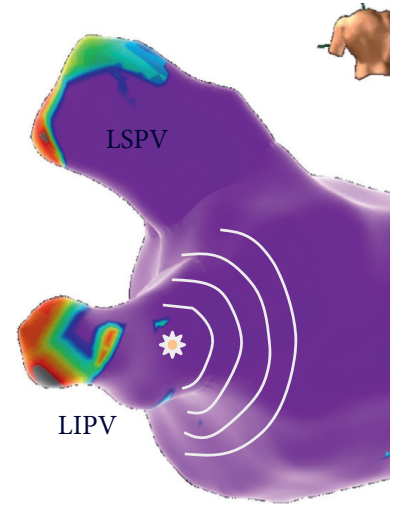

(a)

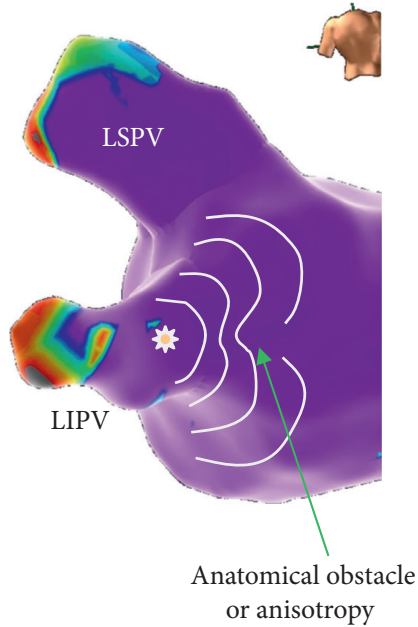

(b)

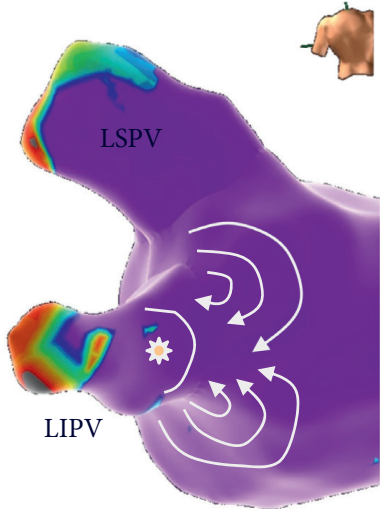

(c)

FIGURE 2: (a) The arrhythmic firing from the left inferior PV propagates normally to the atrial myocardium. (b, c) Special conditions such as conduction anisotropy or anatomical obstacle at the central point of the wavefront can create two waves with rotational propagation, capable of maintaining AF.

In addition, the anatomical structure in the PVs and the adjacent atrial myocardium appear to play an important role in arrhythmogenesis. The arrangement of the muscle fibers in many layers with circumferential and longitudinal direction, the abrupt change in the orientation of the muscle fibers, and the greater wall thickness in specific positions, such as in the interpulmonary areas and in the ridge between the orifices of the left PVs and the mouth of the left atrial appendage, are some of the anatomical reasons that may promote arrhythmogenesis [19]. In addition, the proximity of the epicardial ganglionated plexi with the PV antra is probably responsible for autonomous modification of the atrial electrophysiological milieu [20]. As shown by the study of Lee et al. [21] in which high-density cardiac mapping was performed during cardiac surgery, the areas of PV-LA junction demonstrate marked functional conduction delay and circuitous activation patterns, creating the substrate for reentry. Many times, in clinical practice, we have seen AF start with an automatic firing from a PV.

On the other hand, the concept of extra-PV triggers is gaining ground in understanding the mechanism of AF. An extra-PV trigger is an arrhythmic automatic focus, apart from PVs, which with its rapid firing can initiate AF. For the sake of clarity, we must distinguish the concept of the extra$\mathrm{PV}$ trigger from the extra-PV perpetuator. Obviously, the trigger contributes only to AF provocation, while the 
perpetuator operates during AF supporting its maintenance. Thus, focal or rotational sources outside the area of the PVs occurring during AF cannot be referred to as extra-PV triggers. Probably, a didactic example of a nonclinical extra$\mathrm{PV}$ trigger is the introduction of AF with the extrasystoles caused by a roving catheter in the right atrium (Figure 3 ). It is often reported that revealing and ablating extra-PV triggers can be done systematically during the procedure and this contributes to SR maintenance [22]. It should be noted that during ablation, the demonstration of an extra-PV trigger that really has a causal role in the onset of AF is a laborious process with many difficulties and limitations. It is a laborious process with many difficulties and limitations. Several provocation protocols such as pharmacological challenges (usually isoproterenol), atrial stimulation, and successive AF cardioversions can be used for the elicitation of these arrhythmic foci [22]. An ectopic focus outside PVs to truly represent an $\mathrm{AF}$ trigger must be repetitively exhibited and proven to induce AF (Figure 4). It is unknown whether the mapping and ablation of an ectopic activity that occurs incidentally in SR actually represents a source that could also cause clinical AF. It is also unknown whether and how an arrhythmic focus that appears fleetingly and rarely can be consistently identified during a standard EP study. In this sense, finding areas with characteristics that prove causality in the introduction of AF is rare and difficult. In addition, the lack of repeatability in AF induction makes the process of mapping and exact location of such foci extremely hardworking and time consuming.

\subsection{Rotors, Spiral Waves, and Fibrillatory Conduction.} Recent experimental and clinical data describe specific types of functional reentry called rotors or spiral waves. The rotor is characterized by the rotation of a curved depolarization wavefront around a central point (Figure 5). The propagation velocity of the depolarization wavefront is higher at the periphery of the rotor and lower toward the center. Similarly, the cycle length of the depolarization is higher to the circumference as the frontal margin is further away from the tail of the depolarized area, while toward the center, the curvature of the frontal margin increases and its distance from the tail decreases. Thus, the central point of the rotor has a unique frequency of depolarization and is called phase singularity [23]. The phase singularity point is practically unexcitable and its speed is extremely low, but not zero. This fact permits the movement of the central point and therefore of the entire rotor. This characteristic of the rotor distinguishes it from the entirely theoretical model of the leading cycle [24]. The term spiral waves has often been used to describe the same phenomenon as rotors. However, in electrophysiology, rotors are characterized as drivers, while spiral waves refer to the peripheral propagation of rotor-derived impulses. The term scroll waves actually describes the same event in a three-dimensional model $[25,26]$. The rotor can be formed when a regular impulse collides at an anatomical barrier (myocardial fibrosis) or a functional barrier (anisotropy).

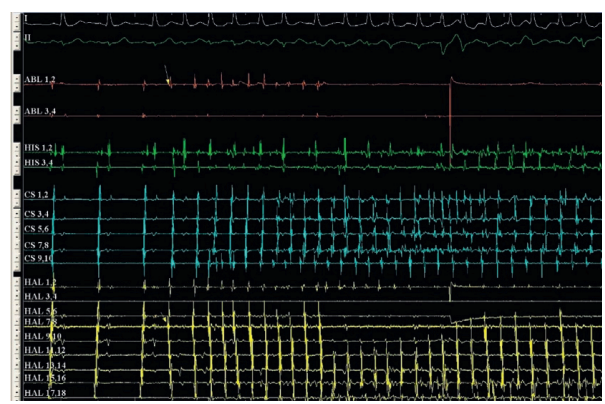

Figure 3: The deflectable 20-polar catheter is placed in the tricuspid annulus in order to perform a linear lesion in the cavotricuspid isthmus. Atrial extrasystoles produced with each movement of the catheters in the right atrium trigger AF.

After its creation, the initial rotor or mother rotor can be broken up into different depolarization waves, which can under appropriate conditions acquire rotor properties, forming the so-called "daughter rotors." Other times, the waves generated by a rotor can propagate with complete disorganization. This is called fibrillatory conduction [17]. The fibrillatory conduction in the presence of rotors was studied in an AF clinical setting with conventional catheters by Atienza et al. [27] and was found to occur when the depolarization frequency becomes higher. It was assumed that the increase in the frequency is caused by the rotor moving to the recording point, generating a Doppler-type effect (Figure 6). It is clear that any point in the atrial myocardium at a given frequency may exhibit the Wenckebach behavior, that is, an apparent conduction delay responsible for the occurrence of fibrillatory conduction. A rapidly firing arrhythmic focus can be spread to the atrial myocardium exhibiting fibrillatory conduction, possibly creating the conditions for AF induction and maintenance (Figure 7). It is possible that the fibrillatory conduction may be also present during macroreentrant circuits, especially with relatively short cycle length. During sequential mapping of unstable atrial tachycardias, we used the selective activation remapping technique, which provided evidence to support the above hypothesis [29] (Figure 8).

1.6. Data from Optical Mapping. In the scientific research of the AF mechanisms beyond theoretical models, conventional mapping, and clinical observations, the optical mapping played a major role. This method sufficiently enriched our knowledge, as we could have high-resolution imaging of the actual electrical impulse during AF. The basic principle of optical mapping is based on the administration of a voltage-sensitive dye, which binds to membrane proteins and has the ability to change its fluorescence when the membrane potential changes during depolarization and repolarization. With appropriately sensitive cameras placed to record the endocardium or the epicardium, this changing in the fluorescence is recorded and so the depolarization and repolarization of the cardiac tissue can be displayed. The obtained recordings undergoing special processing give us a real illustration of the electrical wave propagation [30]. Animal studies have shown that such rotational waves are 


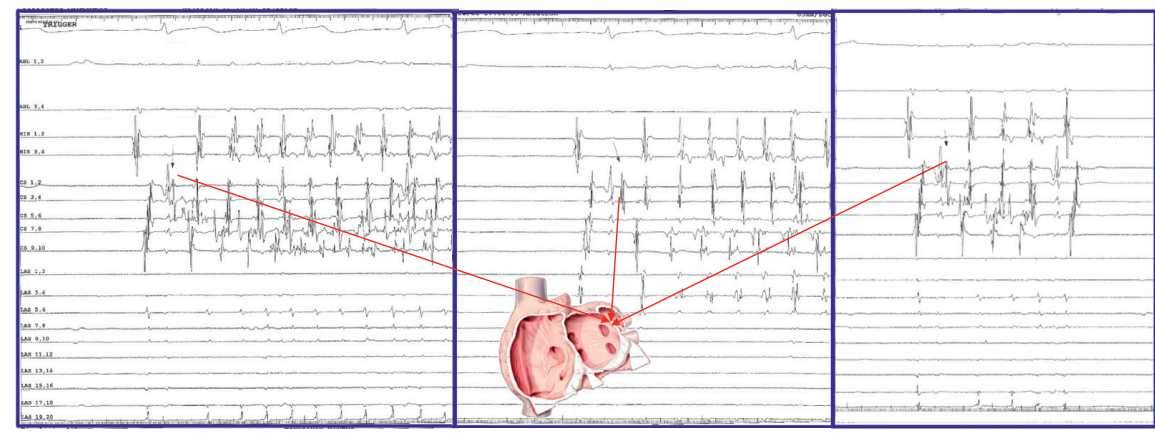

(a)

(b)

(c)

FIGURE 4: An example of the extrapulmonary trigger. In a patient with long-standing persistent AF, after PVI and the electrical cardioversion to $\mathrm{SR}$, a repeated onset of $\operatorname{AF}(\mathrm{a}, \mathrm{b})$ is observed with firing from the same point mapped and ablated in the area between left superior PV and LAA (ligament of Marshall). (c) The firing fails to induce AF. Ablation of this area resulted in the long-term SR maintenance for $>18$ months.
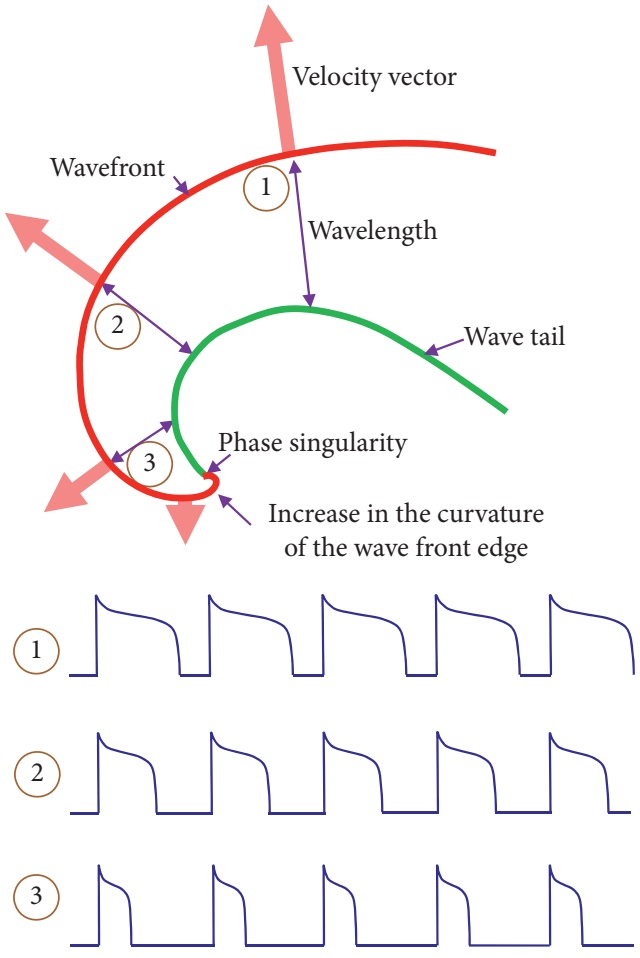

FIGURE 5: Schematic representation of a rotor. The front of the depolarization wave (red line) propagates at a higher speed in the periphery (point 1) than in the center (point 3). The green line represents the repolarized edge. The wavelength, the refractoriness, and the conduction velocity differ from the periphery to the center; however, the depolarization frequency in a stable rotor is the same (see text for details).

maintained during AF over an unexpectedly long period of time. The time of a complete rotation, which by definition represents the cycle length, was extremely short, and the frequency of depolarization was extremely high, leading to the reasonable assumption that the cause of the high frequency in the left atrium is the presence of rotors and spiral waves [31].

Another study of optical mapping performed on human hearts places particular emphasis on the importance of reentry circuits to maintain $\mathrm{AF}$. The study was performed on
8 human hearts, with structural heart disease, removed due to transplantation. The area of the lateral wall of the right atrium was excluded and studied with endocardial and epicardial optical mapping. At the same time, high-resolution magnetic resonance imaging was performed on these atrial segments, which could highlight areas with fibrosis and complex orientation of the myocardial fibers. The mapping revealed multiple micro-reentrant circuits, which were located on the entire thickness of the atrial wall, often having an endocardial and cardiac component. The areas where these circuits were found had a higher rate of fibrosis and significant angulation in the orientation of the myocardial fibers. In fact, ablation in these areas could terminate AF and make it noninducible, unlike other control areas in which ablation did not have such an effect [32].

\section{The Theoretical Knowledge in Clinical Practice}

The revelation of rotors and their importance to AF has led to the development of mapping techniques to reveal this electrical activity in clinical practice.

Focal impulse and rotor modulation (FIRM) is an innovating technique for simultaneous AF mapping developed a few years ago. It utilizes a 64-pole basket catheter of 8 splines containing 8 electrodes each that expands to come into contact with the wall of the left or right atrium, while simultaneously mapping the electrical activity during AF. The analysis of the electrical signals can be presented in a video, displaying the activation and the deactivation of a particular point in alternations of white and black color. In this way, the rotational activity can be depicted and subsequently ablated at the corresponding poles of the catheter. This technique, known as FIRM ablation, had very good results in the CONFIRM (Conventional Ablation for Atrial Fibrillation With or Without Focal Impulse and Rotor Modulation) clinical study [33] (Figure 9).

AF mapping was also attempted with the development of another technology with completely different conception which uses noninvasive body surface mapping. By this technology, the electrical activity is recorded by 252 electrodes in the patient's torso, and after computer processing 


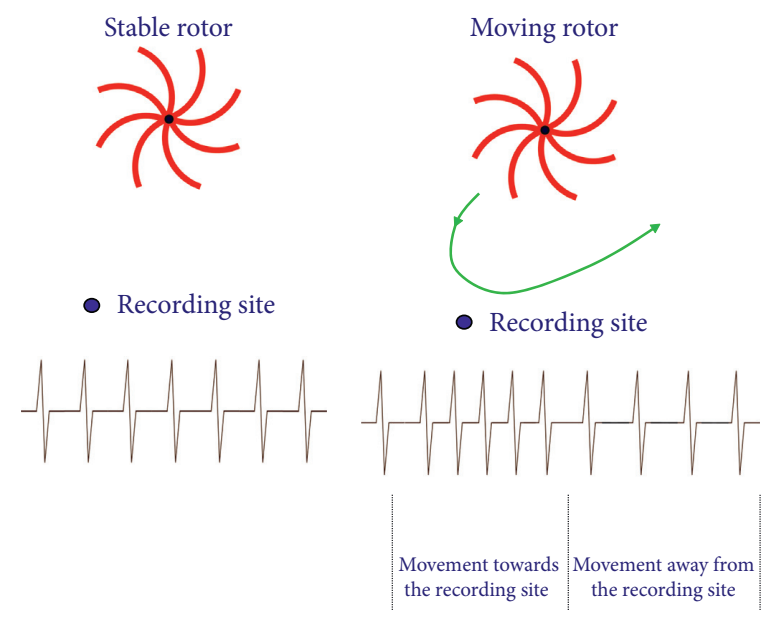

(a)

(b)

FiguRE 6: Doppler-type effect with an increase and decrease in the depolarization frequency when the rotor is moving [28]. (a) Stable rotor. (b) Moving rotor.

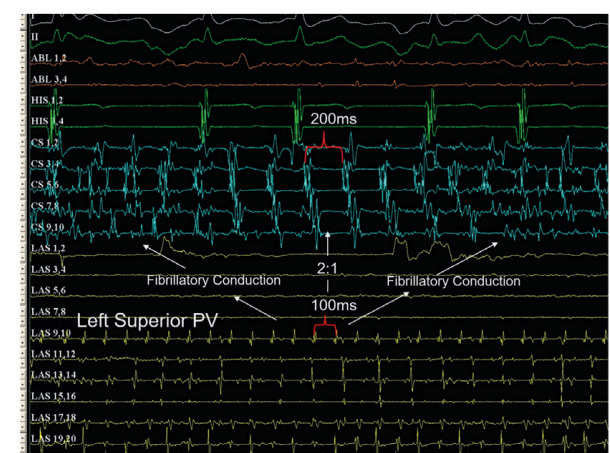

Figure 7: Focal automatic firing (cycle length: $100 \mathrm{~ms}$ ) from LSPV enters the LA either by $2: 1$ conduction or by fibrillatory conduction.

of these recordings, using special mathematical algorithms, the virtual electrical activity is overlaid on the surface of the atria, the three-dimensional model of which has previously been obtained by computed tomography. Thus, we have a simultaneous representation of the electrical activity during AF. Recordings taken with this procedure are also indicative of rotational and focal discharging activity in many areas of both the left and right atrium [34] (Figure 10).

2.1. The Anatomical Substrate of Atrial Fibrillation. The development of AF, even in the absence of other comorbidities, may confer several changes in the atrial myocardium, collectively described as structural remodeling. Very short-term AF produces no ultrastructural alterations, while AF lasting several weeks causes primarily cardiomyocytedependent changes, and long-term persistent AF produces mixed cardiomyocyte- and fibroblast-dependent changes [35]. These alterations in the atrial cardiomyocytes and the myocardial interstitium have been long known to include chronic inflammatory infiltrates, foci of myocyte necrosis, focal replacement fibrosis, and myocyte cytoplasmic vacuoles consistent with myolysis [36]. The degree of such morphological abnormalities, as cellular hypertrophy and myolysis, has been associated with the presence of AF and atrial dilatation [37]. Atrial dilatation in turn, through an increase in atrial substrate size, has been associated with fibrillatory activity maintenance [38]. Moreover, the resulting fibrosis promotes $\mathrm{AF}$ by interrupting fiber bundle continuity and causing local conduction disturbances [39].

In the atrial cardiomyocytes, $\mathrm{AF}$ induces changes in the distribution of proteins responsible for intercellular coupling, the connexins. Heterogeneities in connexin-40 distribution may affect anisotropic conduction in the atria and are known to play a pathophysiological role in paroxysmal AF [40]. In animal models, connexin-40 heterogeneity increases with AF duration and is associated with AF stability [41]. Several cardiac diseases associated with AF, such as hypertension, obesity, hyperglycemia, and heart failure, have been shown to affect the normal distribution of connexins in the atrium, thus causing the conduction disturbances needed for sustaining AF [35, 42].

Another common pathway in the development of $\mathrm{AF}$ persistence in several AF-promoting conditions is atrial fibrosis. Rapid atrial firing promotes fibroblast differentiation to collagen-secreting myofibroblasts through autocrine and paracrine mechanisms, thereby allowing AF to induce atrial fibrosis [43]. Atrial fibrosis occurs earlier in the course of congestive heart failure $(\mathrm{CHF})$ and to a greater extent than in the ventricles. In animal models, CHF-induced atrial fibrosis has been shown to increase the vulnerability to AF and administration of antifibrotic drugs resulted in a reduction of susceptibility to AF [44]. Other cardiac diseases that may lead to atrial fibrosis include obstructive sleep apnea, hypertension, obesity, diabetes, and valvular heart disease, such as mitral stenosis and mitral regurgitation $[45,46]$. In all these cases, the fibrosis has been shown to cause abnormal conduction through the atria, creating a substrate for AF. The mechanism of AF produced by the 


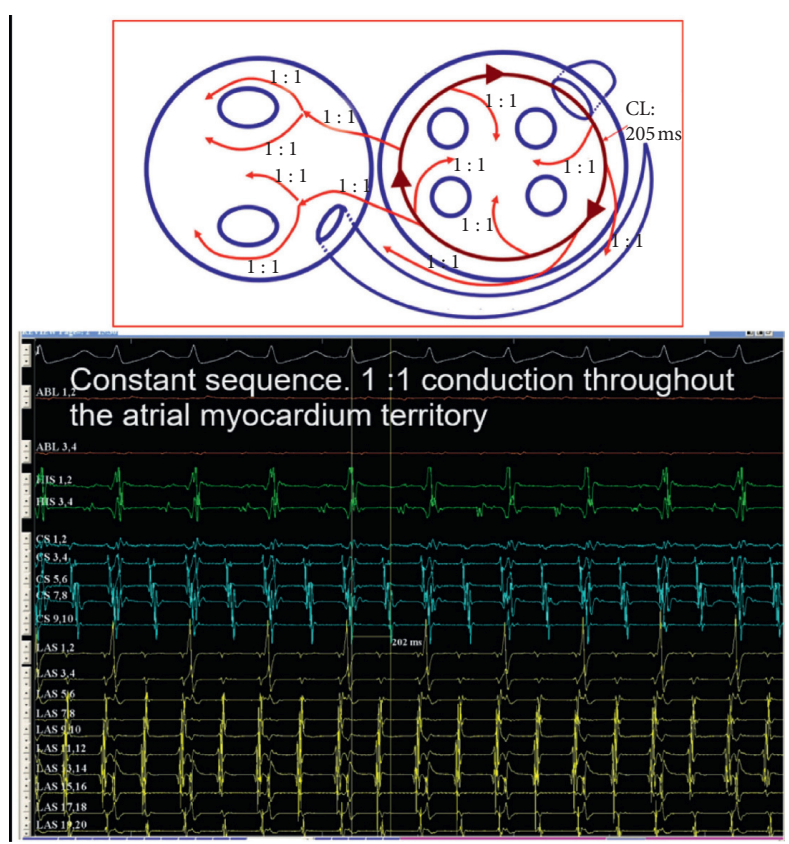

(a)
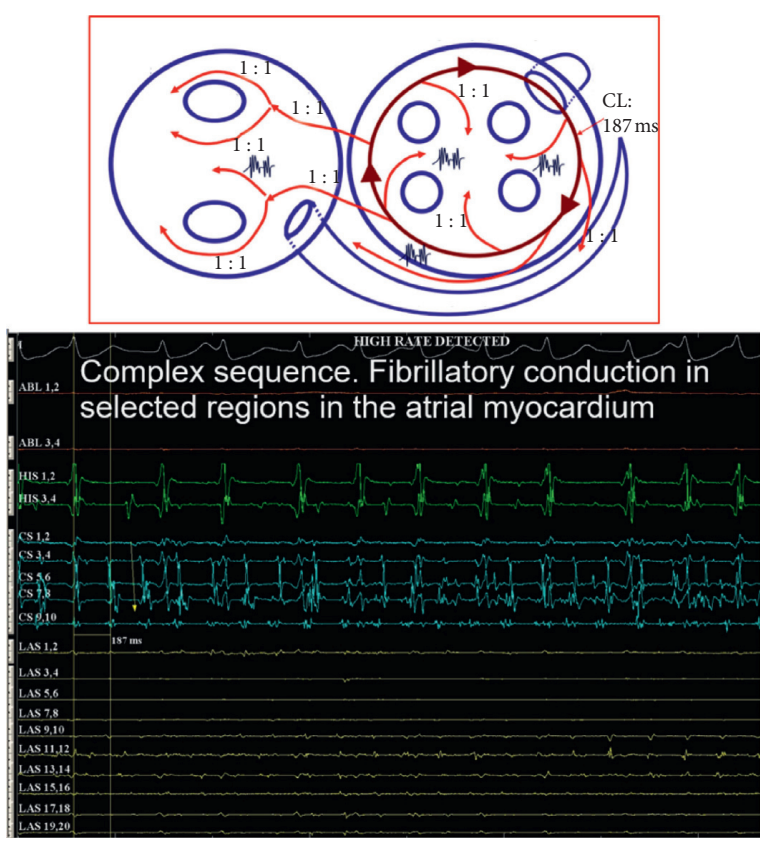

(b)

FIGURE 8: The hypothetical mechanism of the occurrence of fibrillatory conduction in macrocircuits, studied by the technique of selective activation remapping. (a) Clockwise perimitral flutter with $205 \mathrm{~ms} \mathrm{CL}$ and constant $1: 1$ conduction throughout the atria (regular atrial tachycardia). (b) Acceleration of the CL to $187 \mathrm{~ms}$ with fibrillatory conduction (unstable atrial tachycardia) (from Ioannidis et al. [29]).

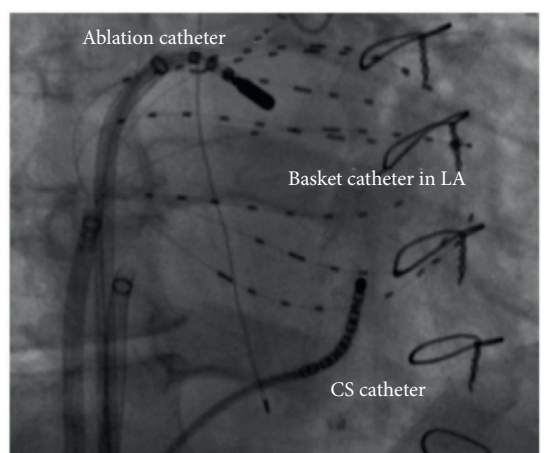

(a)

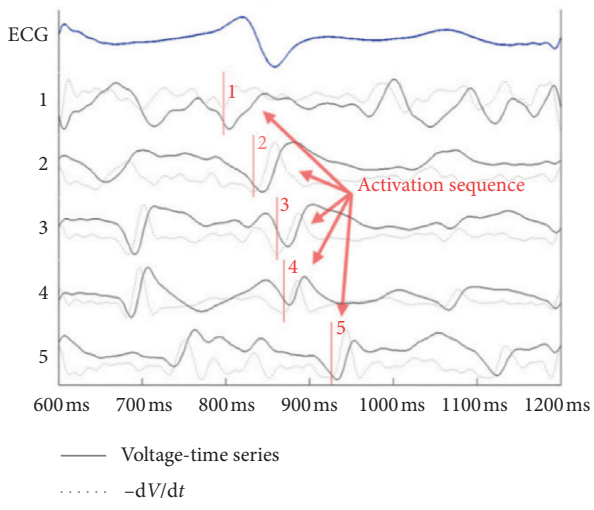

(b)

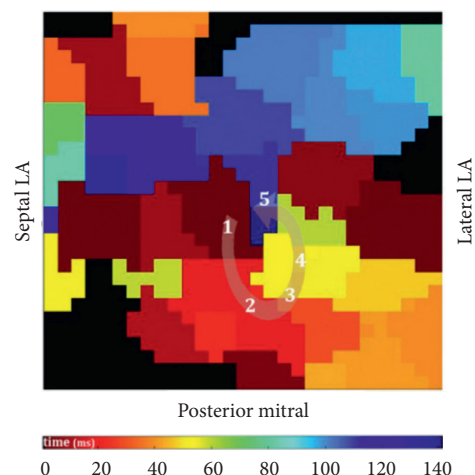

(c)

Figure 9: FIRM mapping. (a) The 64-pole basket catheter in the left atrium. (b) ECG (blue) and unipolar intracardiac signals (black) from the 64-pole basket catheter. (c) Isochronal activation map reconstructed from the corresponding electrograms, illustrating a left atrial rotational activity (from Baykaner et al. [51]).

substrate of atrial fibrosis may include both macroreentry and focal sources that could be originating from RA, LA, or the pulmonary veins [47].

\subsection{Ablation Techniques and Current Capabilities. Over the} years, many ablation techniques have been tested alone or in combination. These included PVI with various means and techniques, the creation of linear lesions [48], with or without confirming bidirectional block, the targeting of complex fractionated atrial electrograms (CFAEs) [49], and the mapping and ablation of ganglionated plexi in order to withdraw the effect of the autonomic nervous system on the genesis of arrhythmia [50,51]. After this long period of time, nowadays, there is a consensus that the wide PVI must necessarily be the first step in ablation. The reasons for PVI effectiveness are many, but probably the common denominator is the exclusion of sufficient atrial myocardium mass of great pathophysiological importance. So today, with the current technology, we can reliably achieve PVI acutely, without, however, being able to guarantee a permanent result. The recurrence of conduction reconnects the previously isolated critical substrate with the rest atrial myocardium, setting thus the conditions for arrhythmia 

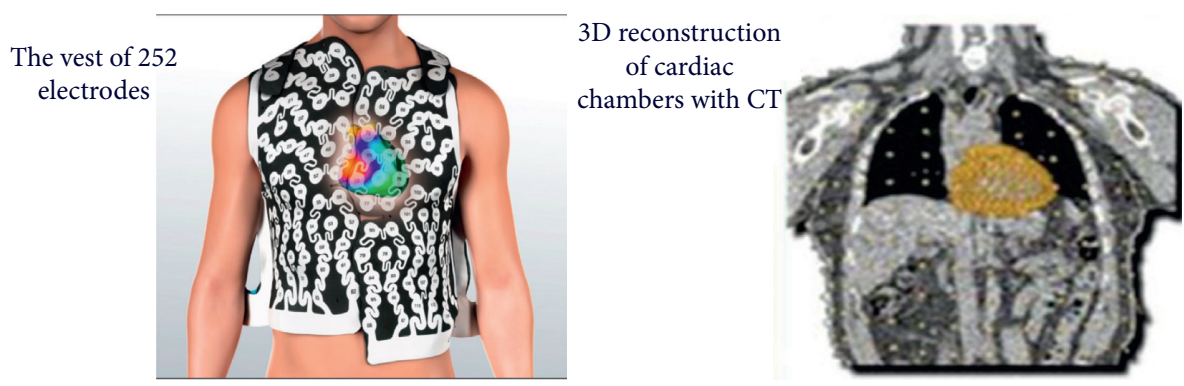

(a)

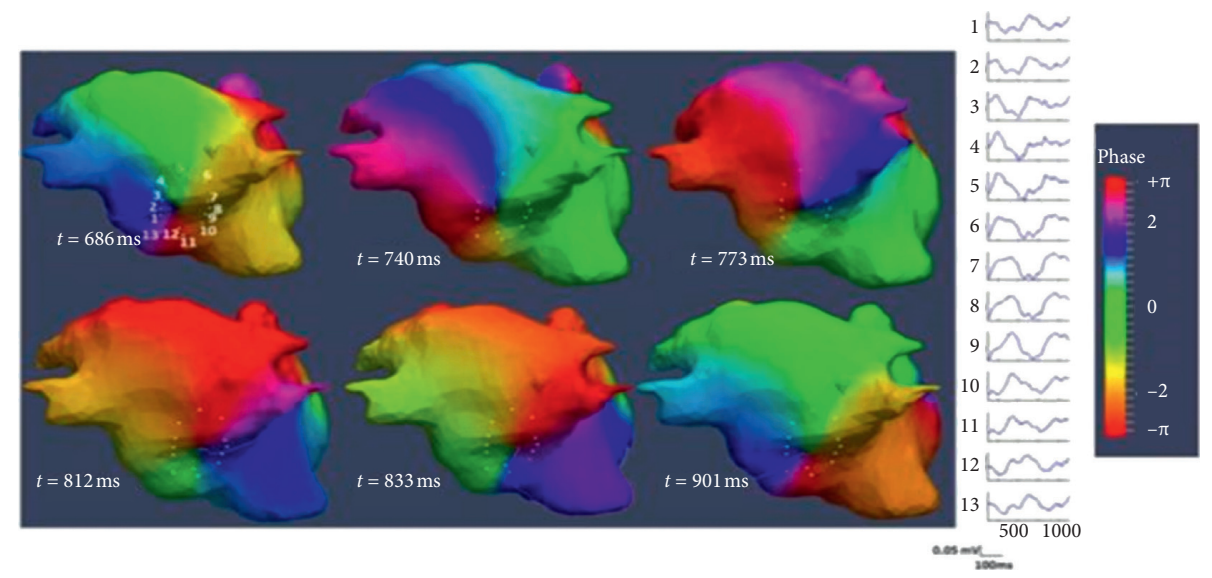

(b)

FIgURE 10: Noninvasive body surface mapping. (a) The vest of 252 electrodes and the three-dimensional reconstruction of cardiac chambers with the computed tomography. The voltage at each point of the torso, taken from the 252 electrodes of the vest, is depicted with virtual electrograms on the surface of the atria. (b) Isochronous maps showing the rotational activity near the antrum of the right inferior PV.

recurrence and the need for reintervention.

In the last years, significant progress has been made in the technical means regarding PVI. Using contact force catheters and the upgraded cryoballoon, more efficient and therefore more durable lesions were achieved [52-54]. In addition, as the large-scale randomized clinical trial "Fire and Ice" showed, the cryoballoon ablation is as effective as RF least for paroxysmal AF [55]. However, from AF cryoablation series, it has become clear that when acute isolation is more convenient, this can guarantee a better clinical outcome [56]. This observation confirms once again the direct correlation of the clinical effectiveness with the interventional effectiveness in PVI.

One of the important questions in the ablation strategy is which technique is most effective in ablating persistent AF. Since by definition persistent AF has a greater pathophysiological dependence on the substrate, it has been suggested that if more intervention is made, in addition to PVI, better results will be achieved. The STAR-AF II trial [57] examined this question and randomly compared PVI vs. PVI plus CFAE ablation vs. PVI plus linear ablation. It appeared that the arms of the additional ablation did not exceed over the PVI-only strategy. This study has complicated our views on the appropriate ablation strategy in persistent AF. However, this led most of the medical community to adopt the opinion that as the first intervention in cases of persistent AF, only PVI should be attempted.

However, even if PVs are completely and permanently isolated, the AF can be maintained either because there are extrapulmonary triggers or because the rest of the atrial substrate is so affected that it can maintain the arrhythmia on its own. Making an assessment of our current knowledge in AF ablation, we would say that although we have made great progress in isolating PVs, in localizing pulmonary and extrapulmonary triggers, we have not made much progress in intervening to the substrate. The ablation of CFAEs, subsidiary to PVI, has shown that it can cause organization or termination of arrhythmia [58-60], but nevertheless, it is completely empirical, without knowing in advance whether they are really critical areas or not [61]. In the study of Ammar-Busch et al., CFAEs were indeed correlated with AF drivers as highlighted by noninvasive body surface mapping. It has been found that CFAEs extend to an average of $50 \%$ in the atrial surface. Although CFAEs can be detected in $75 \%$ of areas with AF drivers, only $25 \%$ of CFAEs correspond to areas with drivers. This means that most CFAEs are noncritical areas for maintaining AF. However, through the process of CFAE ablation, we have clinically proven that in the atrial myocardium all areas are not as important in maintaining AF. Some are more important, and targeted intervention on them can terminate or organize the arrhythmia (Figure 11). So 


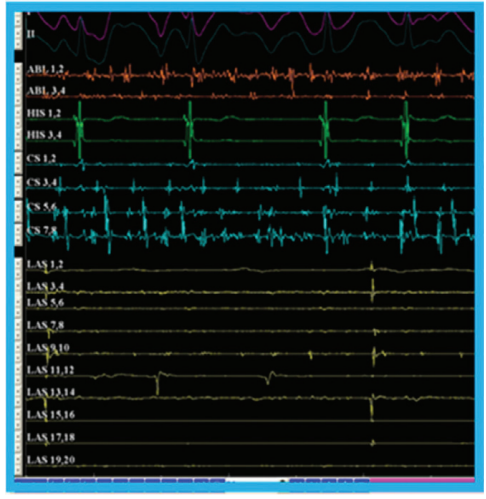

(a)

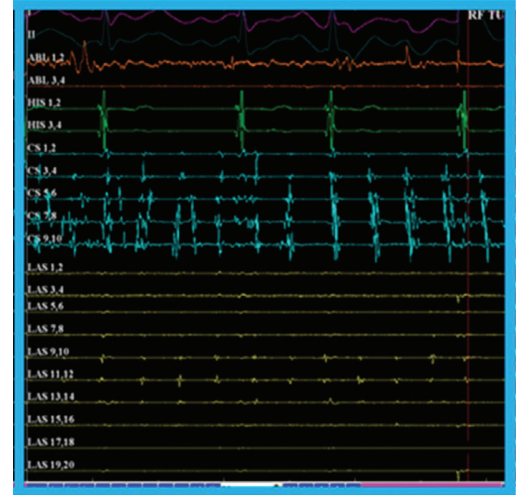

(b)

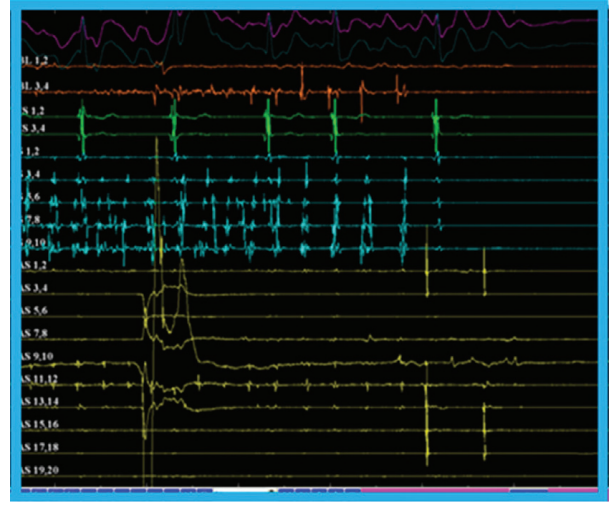

(c)

FIGURE 11: Organization and termination of persistent AF with ablation of CFAEs. (a) Recording of fragmented potentials at the base of the LAA at the onset of ablation. (b) Organization of arrhythmia shortly before termination. (c) Termination of the arrhythmia during ablation in the same area.

came the hypothesis that if we were able to reveal in detail the electrical impulses in AF, we would certainly point out such critical areas susceptible to ablation.

2.3. Targeting Drivers and Rotors. Various efforts have been made to reveal the rotational activity during AF. The theoretical background of these efforts is based on the fact that the detection and ablation of rotors, as experimental studies have shown [32], may also have value in clinical practice. FIRM ablation with selective targeting of areas with repeated rotational or focal activity caused termination or organization of $\mathrm{KM}$ in $86 \%$ of patients with persistent or paroxysmal KM [62]. The initial results of this technique were impressive, as in 9 months of follow-up, in a population with a predominantly persistent $(74 \%) \mathrm{AF}, 82.4 \%$ of patients remained in SR compared with $44.9 \%$ of those who underwent in conventional ablation [33]. In addition, a subanalysis of the CONFIRM trial showed the significance of the elimination of AF rotors and focal sources either directly with system guidance or coincidentally in conventional ablation [63]. However, although the early results of this technique showed impressive results [33, 64-66], more recent studies have failed to confirm the initial encouraging findings [67]. In fact, the recent randomized REAFFIRM trial involving 350 patients with persistent $\mathrm{AF}$ showed that FIRM ablation and subsequent PVI are not superior to PVI only [68].

The use of noninvasive body surface mapping has yielded encouraging results, but it is not yet widely used. The rotational activity is illustrated with virtual maps in the epicardium of the atria. Ablation targets areas with repeatable rotational or focal automatic activity. The greatest advantage of this method is that it is noninvasive. On the contrary, the disadvantages include the difficulty of reproducibility and the ability of dynamic mapping during the procedure, as well as the reliability of the provided maps, as the extension is done only in the epicardium of the sinuses and important areas such as the interatrial septum. However, there is also an issue with the reliability of the isochronal maps, as the projection is made only at the epicardium of the atria, and thus important areas, such as the interatrial septum, are practically hidden. In a clinical study of the system, in 103 patients who underwent $\mathrm{AF}$ ablation, the targeting of the critical areas terminated $\mathrm{AF}$ in $75 \%$ of patients with persistent $\mathrm{AF}$ and in $15 \%$ of patients with longstanding persistent AF. After 12 months' follow-up, 83\% of patients with paroxysmal $\mathrm{AF}$ and $75 \%$ of patients with persistent or long-standing persistent AF remained in SR. The authors claim that the new technique is as effective as the previously used, but that it requires less time [69].

2.4. Future Perspectives. New technologies are being developed to improve the safety and the efficacy, to reduce fluoroscopy and ablation time, and to provide short-learning curve procedures. In addition, one of the issues that new technologies are called upon to solve is the detailed display of the electrical impulse during AF using simultaneous rather than sequential mapping. The AcQMap mapping system (Acutus Medical, CA, USA) using a 10F multipolar spindleshaped catheter can create the three-dimensional model of the atrial chamber by emitting ultrasound waves and then display on the reconstructed surface the propagation of the impulse, using data acquired by measuring the charge density [70] (Figure 12). In the UNCOVER AF study, which was conducted in 13 centers in Europe and Canada, on 127 patients with persistent AF, targeting the rotational activities led to $72 \%$ maintenance of the SR at 12 months after a single procedure and $93 \%$ after 1 or 2 procedures [71].

A critical question that has not yet been clearly answered is whether the direct targeting of AF drivers, beyond the PVs, can ensure better results. For example, if we target the path of the rotor core, will we be able to prevent AF or in this way are we just trying to catch a cloud? On the opposite side of the spectrum, there is the more rough and less electrophysiological technique of atrial compartmentalization by creating strict areas of block (e.g., standard linear lesions). Obviously, we need more data to decide which of the two approaches is preferable or eventually to be led to a combination of them (Figure 13). 


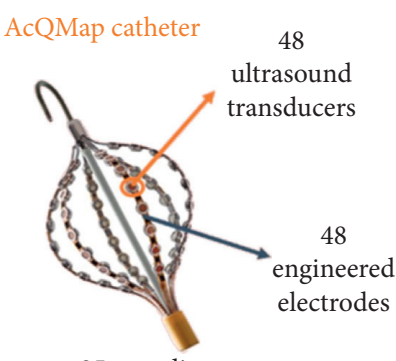

$25 \mathrm{~mm}$ diameter

up to 115,000 ultrasound points/minute 150,000 intracardiac unipolar voltage samples/second
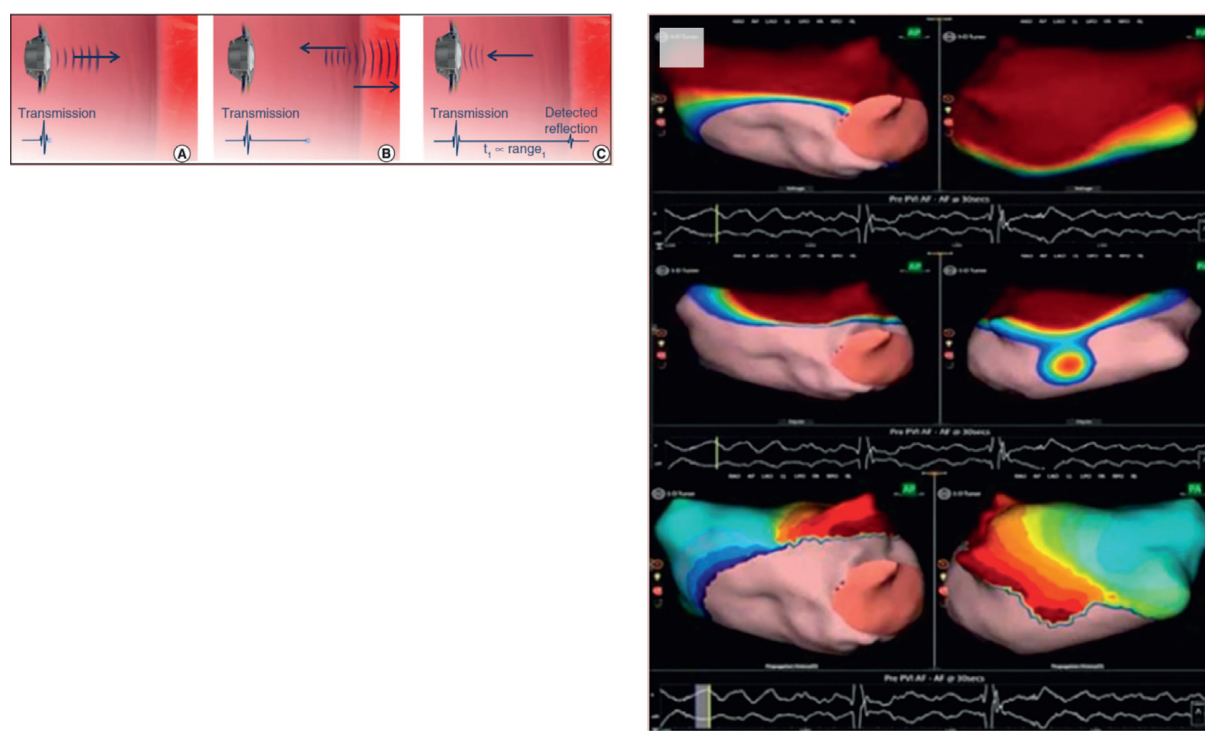

(c)

(b)

(a)

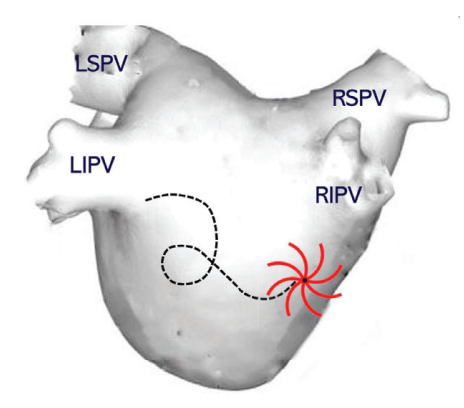

FIgure 12: AcQMap noncontact mapping system (Acutus Medical, CA, USA). (a) The multipolar spherical AcQMap catheter with a diameter of $25 \mathrm{~mm}$ (deployed), which consists of 6 splines, each of which contains 8 electrodes and 8 ultrasound transducers (total 48 ). (b) The three-dimensional model of the LA is constructed by emitting ultrasonic waves reflecting them to the cardiac wall. (c) The system displays on the three-dimensional model the propagation of the impulse, calculated by measuring the dipole density.

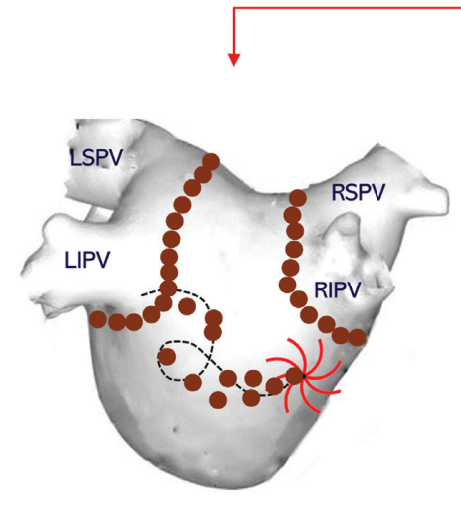

(a)

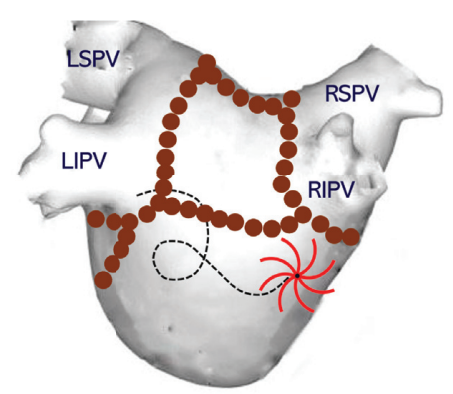

(b)

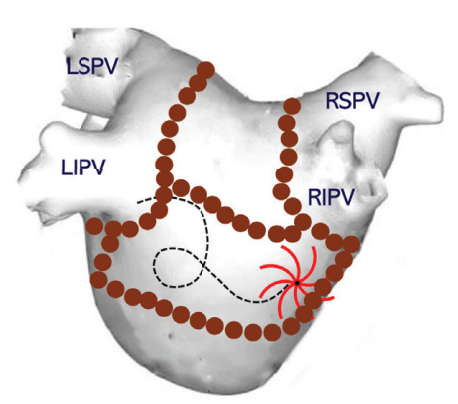

(c)

Figure 13: Possible ablation techniques, beyond PVI, after revealing an AF driver. (a) Direct targeting of the rotor path. (b) Standard linear lesions (box lesion and mitral isthmus block). (c) Linear isolation of the rotor path.

However, in addition to the imaging of the electrophysiological substrate, the detailed imaging of the anatomical substrate would probably be of great help. The location and the extent of atrial fibrosis with late gadolinium enhancement in MRI can give us information that cannot be obtained by any other preinterventional evaluation. The reconstruction of a fibrosis map may guide us in planning the treatment. Nevertheless, from the studies of Marrouche et al. [72], it has been made clear that a high degree of fibrosis is likely a contraindication to conventional ablation 
strategies, as maintaining SR is achieved at a very low rate in the five-year follow-up [73]. Perhaps, on the other hand, it will not be long before the MRI is systematically performed in the electrophysiological laboratory, while at the same time giving the opportunity to visualize the real effect of the therapy [74].

Until today, even if the ablation efficiency in creating transmural lesions has improved, with the use of contact force and cryoballoon catheters, there are still problems in maintaining PV isolation. A new source of energy tested for $\mathrm{AF}$ ablation is the electroporation. By delivering a strong electric field for a very short period of time $(<1 \mathrm{~ms})$, through a simple electrophysiology catheter that is in contact with the myocardium, the lipid bilayer of the cell is specifically disrupted. Multiple microscopic pores are formed in the cell membrane, resulting in disturbance of cell homeostasis and apoptosis. The most important feature of electroporation is that the energy threshold that is necessary to create this irreversible damage is different for different tissues. As a result, ablation can be performed in a very short time in the areas of interest of the myocardium, avoiding damage to neighboring organs such as the esophagus [75]. In a recently published study, Reddy et al. using pulsed field ablation with a "one-shot" multispline catheter conducted successful PVI and LA posterior wall isolation in 25 patients with persistent $\mathrm{AF}$ [76]. It is obvious that current mapping systems are being prepared for changes in the coming years, as their upgrades will integrate new technologies that have proven to be effective and easy to use. For example, it would be beneficial for all 3D mapping systems to add the ability of one-shot PV ablation. This will save time in relation to the time-consuming pointby-point ablation process and will allow further intervention if needed.

\section{Conclusions}

In an era of rapid developments and changes in the field of $\mathrm{AF}$ ablation, it seems that in the coming years the wide PV isolation will remain the cornerstone as ablation strategy. If the new technologies ensure more permanent and safer lesions and allow a detailed depiction of the electrical impulse during $\mathrm{AF}$, then an important step will be taken to definitively treat AF with ablation. The illustration of the electrical activity opens up a new field of research and expectations that we do not know whether it will ultimately be an advantage over current techniques. Of course, we are at the beginning of a journey and the first attempts sometimes have encouraging and sometimes disappointing results. However, the effort to understand the electrophysiology of AF in order to successfully ablate it must not stop, as we seem to be moving toward a more pathophysiological and less empirical approach.

\section{Abbreviations}

AF: Atrial fibrillation

PV: Pulmonary vein

PVI: Pulmonary vein isolation
SR: $\quad$ Sinus rhythm

FIRM: Focal impulse and rotor modulation

CFAEs: Complex fractionated atrial electrograms

LA: Left atrium

CL: Cycle length

LAA: Left atrial appendage

LSPV: Left superior pulmonary vein.

\section{Data Availability}

The data used to support the findings of this study are included within the article.

\section{Conflicts of Interest}

The authors declare that there are no conflicts of interest regarding the publication of this paper.

\section{References}

[1] T. Lewis, The Mechanism and Graphic Registration of the Heart Beat, Shaw \& Sons, London, UK, 1st edition, 1920.

[2] G. K. Moe and J. A. Abildskov, "Atrial fibrillation as a selfsustaining arrhythmia independent of focal discharge," American Heart Journal, vol. 58, no. 1, pp. 59-70, 1959.

[3] G. K. Moe, W. C. Rheinboldt, and J. A. Abildskov, "A computer model of atrial fibrillation," American Heart Journal, vol. 67, no. 2, pp. 200-220, 1964.

[4] M. A. L. W. Allessie, F. I. M. Bonke, and S. J. Hollen, Experimental Evaluation of Moe's Multiple Wavelet Hypothesis of Atrial Fibrillation, Grune \& Stratton, New York, NY, USA, 1985.

[5] Z. Wang, P. Pagé, and S. Nattel, "Mechanism of flecainide's antiarrhythmic action in experimental atrial fibrillation," Circulation Research, vol. 71, no. 2, pp. 271-287, 1992.

[6] J. Wang, G. W. Bourne, Z. Wang, C. Villemaire, M. Talajic, and S. Nattel, "Comparative mechanisms of antiarrhythmic drug action in experimental atrial fibrillation. importance of use-dependent effects on refractoriness," Circulation, vol. 88, no. 3, pp. 1030-1044, 1993.

[7] J. L. Cox, R. B. Schuessler, H. J. D'Agostino et al., "The surgical treatment of atrial fibrillation," The Journal of Thoracic and Cardiovascular Surgery, vol. 101, no. 4, pp. 569-583, 1991.

[8] M. Haïssaguerre, P. Jaï, D. C. Shah et al., "Spontaneous initiation of atrial fibrillation by ectopic beats originating in the pulmonary veins," New England Journal of Medicine, vol. 339, no. 10, pp. 659-666, 1998.

[9] A. Shiroshita-Takeshita, B. J. J. M. Brundel, and S. Nattel, "Atrial fibrillation: basic mechanisms, remodeling and triggers," Journal of Interventional Cardiac Electrophysiology, vol. 13, no. 3, pp. 181-193, 2005.

[10] M. A. Allessie, F. I. Bonke, and F. J. Schopman, "Circus movement in rabbit atrial muscle as a mechanism of tachycardia. III. The "leading circle" concept: a new model of circus movement in cardiac tissue without the involvement of an anatomical obstacle," Circulation Research, vol. 41, no. 1, pp. 9-18, 1977.

[11] T. S. M. Tsang, M. E. Barnes, K. R. Bailey et al., "Left atrial volume: important risk marker of incident atrial fibrillation in 1655 older men and women," Mayo Clinic Proceedings, vol. 76, no. 5, pp. 467-475, 2001. 
[12] P. Papageorgiou, K. Monahan, N. G. Boyle et al., "Site-dependent intra-atrial conduction delay," Circulation, vol. 94, no. 3, pp. 384-389, 1996.

[13] F. G. Cosio, J. Palacios, J. M. Vidal, E. G. Cocina, M. A. Gómez-Sánchez, and L. Tamargo, "Electrophysiologic studies in atrial fibrillation," The American Journal of Cardiology, vol. 51, no. 1, pp. 122-130, 1983.

[14] A. R. Ramdat Misier, T. Opthof, N. M. Van Hemel et al., "Increased dispersion of "refractoriness" in patients with idiopathic paroxysmal atrial fibrillation," Journal of the American College of Cardiology, vol. 19, no. 7, pp. 1531-1535, 1992.

[15] M. Vaquero, D. Calvo, and J. Jalife, "Cardiac fibrillation: from ion channels to rotors in the human heart," Heart Rhythm, vol. 5, no. 6, pp. 872-879, 2008.

[16] M. Mansour, R. Mandapati, O. Berenfeld, J. Chen, F. H. Samie, and J. Jalife, "Left-to-right gradient of atrial frequencies during acute atrial fibrillation in the isolated sheep heart," Circulation, vol. 103, no. 21, pp. 2631-2636, 2001.

[17] J. Jalife, O. Berenfelda, and M. Mansourb, "Mother rotors and fibrillatory conduction: a mechanism of atrial fibrillation," Cardiovascular Research, vol. 54, no. 2, pp. 204-216, 2002.

[18] J. R. Ehrlich, T. J. Cha, L. Zhang et al., "Cellular electrophysiology of canine pulmonary vein cardiomyocytes: action potential and ionic current properties," The Journal of Physiology, vol. 551, no. Pt 3, pp. 801-813, 2003.

[19] S. Y. Ho, J. A. Cabrera, V. H. Tran, J. Farré, R. H. Anderson, and D. Sánchez-Quintana, "Architecture of the pulmonary veins: relevance to radiofrequency ablation," Heart, vol. 86, no. 3, pp. 265-270, 2001.

[20] R. Vaitkevicius, I. Saburkina, K. Rysevaite et al., "Nerve supply of the human pulmonary veins: an anatomical study," Heart Rhythm, vol. 6, no. 2, pp. 221-228, 2009.

[21] G. Lee, S. Spence, A. Teh et al., "High-density epicardial mapping of the pulmonary vein-left atrial junction in humans: insights into mechanisms of pulmonary vein arrhythmogenesis," Heart Rhythm, vol. 9, no. 2, pp. 258-264, 2012.

[22] P. Santangeli and F. E. Marchlinski, "Techniques for the provocation, localization, and ablation of non-pulmonary vein triggers for atrial fibrillation," Heart Rhythm, vol. 14, no. 7, pp. 1087-1096, 2017.

[23] S. V. Pandit and J. Jalife, "Rotors and the dynamics of cardiac fibrillation," Circulation Research, vol. 112, no. 5, pp. 849-862, 2013.

[24] P. Comtois, J. Kneller, and S. Nattel, "Of circles and spirals: bridging the gap between the leading circle and spiral wave concepts of cardiac reentry," Europace, vol. 7, no. Suppl 2, pp. 10-20, 2005.

[25] O. Berenfeld and A. M. Pertsov, "Dynamics of intramural scroll waves in three-dimensional continuous myocardium with rotational anisotropy," Journal of Theoretical Biology, vol. 199, no. 4, pp. 383-394, 1999.

[26] M. Yamazaki, S. Mironov, C. Taravant et al., "Heterogeneous atrial wall thickness and stretch promote scroll waves anchoring during atrial fibrillation," Cardiovascular Research, vol. 94, no. 1, pp. 48-57, 2012.

[27] F. Atienza, D. Calvo, J. Almendral et al., "Mechanisms of fractionated electrograms formation in the posterior left atrium during paroxysmal atrial fibrillation in humans," Journal of the American College of Cardiology, vol. 57, no. 9, pp. 1081-1092, 2011.

[28] M. S. Guillem, A. M. Climent, M. Rodrigo, F. FernándezAvilés, F. Atienza, and O. Berenfeld, "Presence and stability of rotors in atrial fibrillation: evidence and therapeutic implications," Cardiovascular Research, vol. 109, no. 4, pp. $480-492,2016$.

[29] P. Ioannidis, T. Zografos, C. Vassilopoulos et al., "Selective activation re-mapping reveals the mechanism in apparently unstable atrial tachycardias," Journal of Atrial Fibrillation, vol. 11, no. 6, p. 2152, 2019.

[30] D. Lang, M. Sulkin, Q. Lou, and I. R. Efimov, "Optical mapping of action potentials and calcium transients in the mouse heart," Journal of Visualized Experiments, vol. 55, Article ID e3275, 2011.

[31] R. Mandapati, A. Skanes, J. Chen, O. Berenfeld, and J. Jalife, "Stable microreentrant sources as a mechanism of atrial fibrillation in the isolated sheep heart," Circulation, vol. 101, no. 2, pp. 194-199, 2000.

[32] B. J. Hansen, J. Zhao, T. A. Csepe et al., "Atrial fibrillation driven by micro-anatomic intramural re-entry revealed by simultaneous sub-epicardial and sub-endocardial optical mapping in explanted human hearts," European Heart Journal, vol. 36, no. 35, pp. 2390-2401, 2015.

[33] S. M. Narayan, D. E. Krummen, K. Shivkumar, P. Clopton, W.-J. Rappel, and J. M. Miller, "Treatment of atrial fibrillation by the ablation of localized sources," Journal of the American College of Cardiology, vol. 60, no. 7, pp. 628-636, 2012.

[34] M. Haissaguerre, M. Hocini, A. J. Shah et al., "Noninvasive panoramic mapping of human atrial fibrillation mechanisms: a feasibility report," Journal of Cardiovascular Electrophysiology, vol. 24, no. 6, pp. 711-717, 2013.

[35] A. Goette, J. M. Kalman, L. Aguinaga et al., "EHRA/HRS/ APHRS/SOLAECE expert consensus on atrial cardiomyopathies: definition, characterization, and clinical implication," Europace, vol. 18, no. 10, pp. 1455-1490, 2016.

[36] A. Frustaci, C. Chimenti, F. Bellocci, E. Morgante, M. A. Russo, and A. Maseri, "Histological substrate of atrial biopsies in patients with lone atrial fibrillation," Circulation, vol. 96, no. 4, pp. 1180-1184, 1997.

[37] L. Mary-Rabine, A. Albert, T. D. Pham et al., "The relationship of human atrial cellular electrophysiology to clinical function and ultrastructure," Circulation Research, vol. 52, no. 2, pp. 188-199, 1983.

[38] R. Zou, J. Kneller, L. J. Leon, and S. Nattel, "Substrate size as a determinant of fibrillatory activity maintenance in a mathematical model of canine atrium," American Journal of Physiology-Heart and Circulatory Physiology, vol. 289, no. 3, pp. H1002-H1012, 2005.

[39] S. Nattel and M. Harada, "Atrial remodeling and atrial fibrillation," Journal of the American College of Cardiology, vol. 63, no. 22, pp. 2335-2345, 2014.

[40] J. Gemel, A. E. Levy, A. R. Simon et al., "Connexin40 abnormalities and atrial fibrillation in the human heart," Journal of Molecular and Cellular Cardiology, vol. 76, pp. 159-168, 2014.

[41] H. Van Der Velden, J. Ausma, M. B. Rook et al., "Gap junctional remodeling in relation to stabilization of atrial fibrillation in the goat," Cardiovascular Research, vol. 46, no. 3, pp. 476-486, 2000.

[42] N. J. Severs, A. F. Bruce, E. Dupont, and S. Rothery, "Remodelling of gap junctions and connexin expression in diseased myocardium," Cardiovascular Research, vol. 80, no. 1, pp. 9-19, 2008.

[43] D. W. Kitzman and W. D. Edwards, "Age-related changes in the anatomy of the normal human heart," Journal of Gerontology, vol. 45, no. 2, pp. M33-M39, 1990.

[44] K. W. Lee, T. H. Everett, D. Rahmutula et al., "Pirfenidone prevents the development of a vulnerable substrate for atrial 
fibrillation in a canine model of heart failure," Circulation, vol. 114, no. 16, pp. 1703-1712, 2006.

[45] A. Boldt, U. Wetzel, J. Lauschke et al., "Fibrosis in left atrial tissue of patients with atrial fibrillation with and without underlying mitral valve disease," Heart, vol. 90, no. 4, pp. 400-405, 2004.

[46] M.-C. Chen, J.-P. Chang, S.-C. Huang et al., "Dedifferentiation of atrial cardiomyocytes in cardiac valve disease: unrelated to atrial fibrillation," Cardiovascular Pathology, vol. 17, no. 3, pp. 156-165, 2008.

[47] T. H. Everett and J. E. Olgin, "Atrial fibrosis and the mechanisms of atrial fibrillation," Heart Rhythm, vol. 4, no. 3, pp. S24-S27, 2007.

[48] S. Knecht, M. Hocini, M. Wright et al., "Left atrial linear lesions are required for successful treatment of persistent atrial fibrillation," European Heart Journal, vol. 29, no. 19, pp. 2359-2366, 2008.

[49] K. Nademanee, J. McKenzie, E. Kosar et al., "A new approach for catheter ablation of atrial fibrillation: mapping of the electrophysiologic substrate," Journal of the American College of Cardiology, vol. 43, no. 11, pp. 2044-2053, 2004.

[50] D. G. Katritsis, E. Giazitzoglou, T. Zografos, E. Pokushalov, S. S. Po, and A. J. Camm, "Rapid pulmonary vein isolation combined with autonomic ganglia modification: a randomized study," Heart Rhythm, vol. 8, no. 5, pp. 672-678, 2011.

[51] T. Baykaner, T. A. Zografos, J. A. B. Zaman et al., "Spatial relationship of organized rotational and focal sources in human atrial fibrillation to autonomic ganglionated plexi," International Journal of Cardiology, vol. 240, pp. 234-239, 2017.

[52] A. Aryana, M. R. Bowers, P. G. O’Neill et al., "Outcomes of cryoballoon ablation of atrial fibrillation: a comprehensive review," Journal of Atrial Fibrillation, vol. 8, p. 1231, 2015.

[53] S. Miyazaki, H. Taniguchi, H. Hachiya et al., "Durability of cryothermal pulmonary vein isolation-creating contiguous lesions is necessary for persistent isolation," International Journal of Cardiology, vol. 220, pp. 395-399, 2016.

[54] J. Kautzner, P. Neuzil, H. Lambert et al., "Efficas II: optimization of catheter contact force improves outcome of pulmonary vein isolation for paroxysmal atrial fibrillation," Europace, vol. 17, no. 8, pp. 1229-1235, 2015.

[55] K.-H. Kuck, J. Brugada, A. Fürnkranz et al., "Cryoballoon or radiofrequency ablation for paroxysmal atrial fibrillation," New England Journal of Medicine, vol. 374, no. 23, pp. 2235-2245, 2016.

[56] K. R. J. Chun, M. Stich, A. Fürnkranz et al., "Individualized cryoballoon energy pulmonary vein isolation guided by realtime pulmonary vein recordings, the randomized ICE-T trial," Heart Rhythm, vol. 14, no. 4, pp. 495-500, 2017.

[57] A. Verma, C.-Y. Jiang, T. R. Betts et al., "Approaches to catheter ablation for persistent atrial fibrillation," New England Journal of Medicine, vol. 372, no. 19, pp. 1812-1822, 2015.

[58] C. S. Elayi, A. Verma, L. Di Biase et al., "Ablation for longstanding permanent atrial fibrillation: results from a randomized study comparing three different strategies," Heart Rhythm, vol. 5, no. 12, pp. 1658-1664, 2008.

[59] W.-J. Li, Y.-Y. Bai, H.-Y. Zhang et al., "Additional ablation of complex fractionated atrial electrograms after pulmonary vein isolation in patients with atrial fibrillation," Circulation: Arrhythmia and Electrophysiology, vol. 4, no. 2, pp. 143-148, 2011.

[60] R. M. Hayward, G. A. Upadhyay, T. Mela et al., "Pulmonary vein isolation with complex fractionated atrial electrogram ablation for paroxysmal and nonparoxysmal atrial fibrillation: a meta-analysis," Heart Rhythm, vol. 8, no. 7, pp. 994-1000, 2011.

[61] N. Navoret, S. Jacquir, G. Laurent, and S. Binczak, "Detection of complex fractionated atrial electrograms using recurrence quantification analysis," IEEE Transactions on Biomedical Engineering, vol. 60, no. 7, pp. 1975-1982, 2013.

[62] S. M. Narayan, K. Shivkumar, D. E. Krummen, J. M. Miller, and W.-J. Rappel, "Panoramic electrophysiological mapping but not electrogram morphology identifies stable sources for human atrial fibrillation," Circulation: Arrhythmia and Electrophysiology, vol. 6, no. 1, pp. 58-67, 2013.

[63] S. M. Narayan, D. E. Krummen, P. Clopton, K. Shivkumar, and J. M. Miller, "Direct or coincidental elimination of stable rotors or focal sources may explain successful atrial fibrillation ablation," Journal of the American College of Cardiology, vol. 62, no. 2, pp. 138-147, 2013.

[64] C. Gianni, S. Mohanty, L. Di Biase et al., "Acute and early outcomes of focal impulse and rotor modulation (FIRM)guided rotors-only ablation in patients with nonparoxysmal atrial fibrillation," Heart Rhythm, vol. 13, no. 4, pp. 830-835, 2016.

[65] S. M. Narayan and J. A. B. Zaman, "Mechanistically based mapping of human cardiac fibrillation," The Journal of Physiology, vol. 594, no. 9, pp. 2399-2415, 2016.

[66] P. Sommer, S. Kircher, S. Rolf et al., "Successful repeat catheter ablation of recurrent longstanding persistent atrial fibrillation with rotor elimination as the procedural endpoint: a case series," Journal of Cardiovascular Electrophysiology, vol. 27, no. 3, pp. 274-280, 2016.

[67] E. Buch, M. Share, R. Tung et al., "Long-term clinical outcomes of focal impulse and rotor modulation for treatment of atrial fibrillation: a multicenter experience," Heart Rhythm, vol. 13, pp. 636-641, 2016.

[68] J. Brachmann, J. Hummel, D. Wilber et al., "Prospective randomized comparison of rotor ablation vs. conventional ablation for treatment of persistent atrial fibrillation-the REAFFIRM trial," in Proceedings of the Heart Rhythm Society 40th Annual Scientific Sessions, San Francisco, CA, USA, May 2019.

[69] M. Haissaguerre, M. Hocini, A. Denis et al., "Driver domains in persistent atrial fibrillation," Circulation, vol. 130, no. 7 , pp. 530-538, 2014.

[70] R. Shi, M. Norman, Z. Chen, and T. Wong, "Individualized ablation strategy guided by live simultaneous global mapping to treat persistent atrial fibrillation," Future Cardiology, vol. 14, no. 3, pp. 237-249, 2018.

[71] S. Willems, A. Verma, T. R. Betts et al., "Targeting nonpulmonary vein sources in persistent atrial fibrillation identified by noncontact charge density mapping," Circulation: Arrhythmia and Electrophysiology, vol. 12, no. 7, Article ID e007233, 2019.

[72] N. F. Marrouche, D. Wilber, G. Hindricks et al., "Association of atrial tissue fibrosis identified by delayed enhancement MRI and atrial fibrillation catheter ablation," JAMA, vol. 311, no. 5, pp. 498-506, 2014.

[73] M. G. Chelu, J. B. King, E. G. Kholmovski et al., "Atrial fibrosis by late gadolinium enhancement magnetic resonance imaging and catheter ablation of atrial fibrillation: 5-year follow-up data," Journal of the American Heart Association, vol. 7, no. 23, Article ID e006313, 2018.

[74] I. Paetsch, P. Sommer, C. Jahnke et al., "Clinical workflow and applicability of electrophysiological cardiovascular magnetic resonance-guided radiofrequency ablation of isthmus- 
dependent atrial flutter," European Heart Journal-Cardiovascular Imaging, vol. 20, no. 2, pp. 147-156, 2019.

[75] F. H. M. Wittkampf, R. van Es, and K. Neven, "Electroporation and its relevance for cardiac catheter ablation," JACC: Clinical Electrophysiology, vol. 4, no. 8, pp. 977-986, 2018.

[76] V. Y. Reddy, A. Anic, J. Koruth et al., "Pulsed field ablation in patients with persistent atrial fibrillation," Journal of the American College of Cardiology, vol. 76, no. 9, pp. 1068-1080, 2020 . 